\title{
Mid-Infrared Ge-on-Si Electro-absorption Modulator
}

\author{
Tiantian Li ${ }^{1,2}$, Milos Nedeljkovic ${ }^{1}$, Nannicha Hattasan ${ }^{1}$, Ali Z. Khokhar ${ }^{1}$, Scott A. Reynolds ${ }^{1}$, Stevan \\ Stanković $^{1}$, Mehdi Banakar ${ }^{1}$, Wei Cao ${ }^{1}$, Zhibo Qu ${ }^{1}$, Callum G. Littlejohns ${ }^{1}$, Jordi Soler Penades ${ }^{1}$, Katarzyina \\ Grabska $^{1}$, Lorenzo Mastronardi ${ }^{1}$, David J. Thomson ${ }^{1}$, Frederic Y. Gardes ${ }^{1}$, Graham T. Reed ${ }^{1}$, Hequan Wu ${ }^{2}$, \\ Zhiping Zhou ${ }^{2}$ and Goran Z. Mashanovich ${ }^{1, *}$ \\ ${ }^{1}$ Optoelectronics Research Centre, University of Southampton, Southampton SO17 1BJ, U.K. \\ ${ }^{2}$ State Key Laboratory of Advanced Optical Communication Systems and Networks, School of Electronics Engineering and Computer Science, \\ Peking University, Beijing, China 100871 \\ *g.mashanovich@ soton.ac.uk
}

\begin{abstract}
We present the first waveguide electro-absorption modulator in germanium-onsilicon material platform at $3.8 \mu \mathrm{m}$ wavelength, based on free-carrier injection into a straight waveguide. The fabricated $1 \mathrm{~mm}$ long device has modulation depth of $>35 \mathrm{~dB}$ at $7 \mathrm{~V}$.
\end{abstract}

\section{INTRODUCTION}

The mid-infrared (MIR) wavelength range (3-14 $\mu \mathrm{m})$ is attracting considerable attention for spectroscopic sensing applications, because many gases, chemical and biological molecules have strong, unique, absorption bands in the MIR, and because the 3-5 $\mu \mathrm{m}$ and 8-14 $\mu \mathrm{m}$ ranges are also atmospheric transmission windows, which could be exploited for free-space communications. Photonic integration is promising for shrinking the cost and size of existing MIR sensing and communication systems. Siliconon-Insulator (SOI) is already a popular platform for optical communication because of its compatibility with CMOS technology. However, SOI's underlying buried oxide layer has large absorption at longer wavelengths [1]. Germanium-on-silicon (Ge-on-Si) is a promising alternative material platform, because $\mathrm{Ge}$ has low absorption from $2 \mu \mathrm{m}$ to $15 \mu \mathrm{m}$, and the $\mathrm{Ge}$ on-Si platform could have low loss all the way up to $14 \mu \mathrm{m}$ [1]. Ge-on-Si waveguides [2], multimode interferometers [2], grating couplers [2], multiplexers $[3,4]$ and thermo-optic modulators [5] have been reported in the last few years, but higher speed modulation has not yet been addressed.

Due to the centrosymmetric crystal structure of Ge, the Kerr and Pockels effects are weak, while the Franz-Keldysh effect is also negligible for wavelengths longer than $\sim 1.6 \mu \mathrm{m}$. The free-carrier plasma effect of $\mathrm{Ge}$ has been numerically calculated in [6]. It was predicted that Ge modulators exploiting free-carrier absorption should be almost 4 times more effective than in $\mathrm{Si}$ at $3.8 \mu \mathrm{m}$ [6]. In this paper we demonstrate the first MIR Ge-on-Si electro-optic modulator based on the $\mathrm{Ge}$ free-carrier electro- absorption effect, at a wavelength of $3.8 \mu \mathrm{m}$.

\section{DEVICE DESCRIPTION}

The device uses a PIN junction to inject carriers into a rib waveguide structure. Fig. 1(a) shows an optical microscope picture of the device, and Fig. 1(b) shows a schematic of its cross-section. The electrooptical interaction region is $1 \mathrm{~mm}$ long. The thickness of the Ge layer is $3 \mu \mathrm{m}$ and the waveguide width is $2.7 \mu \mathrm{m}$, with a $1.2 \mu \mathrm{m}$ thick slab. The heavy doping regions are $6 \mu \mathrm{m}$ away from the edge of the rib waveguide which takes into account both the absorption loss caused by the high doping regions and the speed of the device. A grating coupler [2] is employed to couple light in and out of the device (Fig. 1(a)).

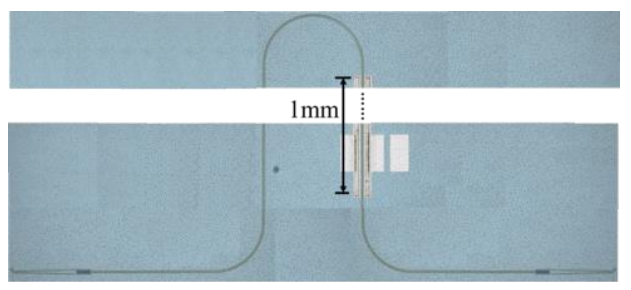

(a)

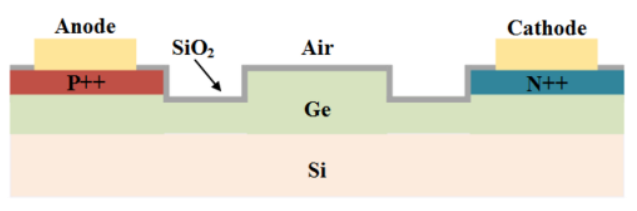

(b)

Fig. 1. (a) Optical microscope image of the device; (b) cross-section diagram of the PIN junction.

Chip fabrication was carried out at the Southampton Nanofabrication Centre, University of Southampton. The gratings and waveguides were defined using e-beam lithography, followed by ICP etching. $1.5 \mu \mathrm{m}$ thick $\mathrm{SiO}_{2}$ was employed as a hard mask for ion implantation, and was patterned using HF wet etching. The waveguides were etched after 
ion implantation, in order to avoid potential issues arising from non-uniform implant mask thicknesses due to the large etched waveguide step. The ion implantation was conducted at the Surrey Ion Beam Center, University of Surrey. Chain implantations of boron and phosphorous ions were conducted to realize both a high concentration at the surface for good Ohmic contact and doping depths of $500 \mathrm{~nm}$. A $100 \mathrm{~nm} \mathrm{SiO}{ }_{2}$ layer was deposited by PECVD before passivation and metallization, and vias were etched through the $\mathrm{SiO}_{2} .100 \mathrm{~nm}$ thick aluminum electrodes were deposited by e-beam evaporator (LAB700) and patterned using the lift-off technique.

\section{MEASUREMENTS}

The device was characterized using the setup described in [7]. Passive waveguides fabricated on the wafer exhibited non-uniform insertion losses, most likely due to "time dependent haze" defects visible on the Ge material surface, which made it impossible to accurately measure the passive insertion losses of the modulators. According to our initial measurements, the loss of the $1 \mathrm{~mm}$ device should be less than $13.5 \mathrm{~dB}$.

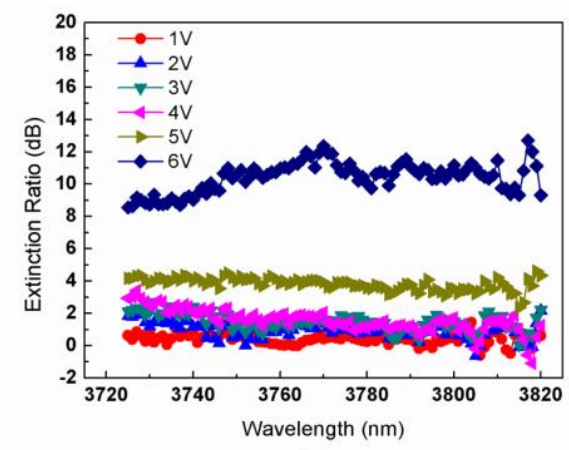

(a)

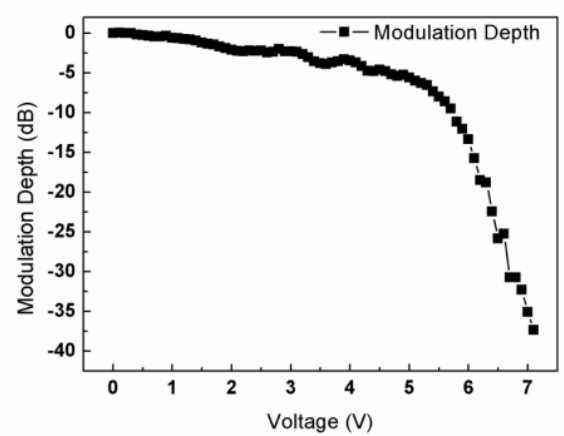

(b)

Fig. 2. (a) The extinction ratio under different voltage from $3720 \mathrm{~nm}$ to $3820 \mathrm{~nm}$; (b) The modulation depth of the device at $3765 \mathrm{~nm}$ under the DC voltage from $0 \mathrm{~V}$ to $7 \mathrm{~V}$.

Fig. 2(a) shows the extinction ratio of the device over the 3720-3820 $\mathrm{nm}$ wavelength range, when different DC bias voltages were applied. The measured wavelength was limited by the laser transmission range and grating coupler bandwidth. However, the modulator was intrinsically limited only by the waveguide bandwidth, since free-carrier absorption in Ge is very strong throughout the MIR. Fig. 2(b) shows the modulation depth of the device under DC voltages from $0 \mathrm{~V}$ to $7 \mathrm{~V}$ at $3765 \mathrm{~nm}$. The modulation depth reaches $35 \mathrm{~dB}$ when $7 \mathrm{~V}$ is applied. Initial characterization of the AC performance of the device, when the modulator was driven by a square wave $7 \mathrm{~V}_{\mathrm{pp}}$ signal with a DC bias of $+3.5 \mathrm{~V}$, showed that the bandwidth is at least $6 \mathrm{MHz}$. The AC measurement is presently limited by the bandwidth of the signal generator used to generate the signal. In addition the bandwidth could be further improved by designing a device with reduced separation between the high doping region and the edge of the rib waveguide.

\section{CONCLUSION}

We have successfully demonstrated the first waveguide based electro-optic modulator at $3.8 \mu \mathrm{m}$, in which the free-carrier electro-absorption effect of $\mathrm{Ge}$ is exploited. The device shows good performance from $3720 \mathrm{~nm}$ to $3820 \mathrm{~nm}$. The working wavelength range could be wider if an end-fire coupling scheme is used. When the applied DC voltage is $7 \mathrm{~V}$, the modulation depth of the $1 \mathrm{~mm}$ long device reaches 35 dB. $6 \mathrm{MHz}$ dynamic operation was also demonstrated under a driving voltage of $7 \mathrm{~V}_{\mathrm{pp}}$ with a DC bias of 3.5 V.

\section{ACKNOWLEDGEMENT}

This work was funded by the EPSRC under the EPSRC MIGRATION project (EP/L01162X/1). Tiantian Li would like to acknowledge the support from the China Scholarship Council.

\section{REFERENCE}

[1] R. Soref, "Mid-infrared photonics in silicon and germanium," Nat. Photonics, vol. 4, no. 8, pp. 495-497, 2010.

[2] M. Nedeljkovic, et al., "Surface grating coupled low loss Geon-Si rib waveguides and multimode interferometers," IEEE Photon. Technol. Lett., vol. 27, no. 10, pp. 1040-1043, 2015. [3] A. Malik, et al., "Germanium-on-silicon mid-infrared arrayed waveguide grating multiplexers," IEEE Photon. Technol. Lett., vol. 25, no. 18, pp. 1805-1808, 2013.

[4] A. Malik, et al., "Germanium-on-silicon planar concave grating wavelength (de)multiplexers in the mid-infrared," Appl. Phys. Lett., vol. 103, no. 16, pp. 161119-1-161119-4, 2013.

[5] A. Malik, et al., "Ge-on-Si and Ge-on-SOI thermo-optic phase shifters for the mid-infrared," Opt. Express, vol. 22, no.23, pp. 28479-28488, 2014.

[6] M. Nedeljkovic, et al., "Predictions of free-carrier electroabsorption and electrorefraction in Germanium," IEEE Photonics J., vol. 7, no. 3, pp. 2600214, 2015.

[7] M. Nedeljkovic, et al., "Mid-infrared thermo-optic modulators in SOI," IEEE Photon. Technol. Lett., vol. 26, no. 13, pp. 13521355, 2014. 